\title{
A NOTE ON'FIXED POINT FREE INVOLUTIONS AND EQUIVARIANT MAPS
}

\author{
JACK UCCI
}

\begin{abstract}
The space $P\left(S^{n}\right)$ of all paths $\omega$ in $S^{n}$ with given initial point $x$ and endpoint $-x$ admits an involution $(T \omega)(t)=$ $-\omega(1-t)$. With the standard antipodal involution on $S^{n-1}$ an equivariant map $P\left(S^{n}\right) \rightarrow S^{n-1}$ is constructed for $n=2,4$, or 8 .
\end{abstract}

A fixed point free involution on a space $X$ is a map $T: X \rightarrow X$ satisfying $T^{2}=$ identity and $T x \neq x$ for every $x \in X$. Three examples of interest are (i) $\left(S^{n}, T_{1}\right)$ with $T_{1} x=-x$; (ii) $\left(V\left(S^{n}\right), T_{2}\right)$ where $V\left(X^{n}\right)=$ the unit tangent sphere bundle of $S^{n}$ and $T_{2}=$ the antipodal action on each fibre; and (iii) $\left(P\left(S^{n}\right), T_{3}\right)$ where $P\left(S^{n}\right)=$ the space of paths with given initial point $x \in S^{n}$ and endpoint $-x$, and $\left(T_{3} \omega\right)(t)=-\omega(1-t)$.

For $(X, T)$ a fixed point free involution the co-index of $X$ is the least integer $n$ for which there exists an equivariant map $(X, T) \rightarrow\left(S^{n}, T_{1}\right)$. A classical result of Borsuk [1] asserts that the co-index of $\left(S^{n}, T_{1}\right)$ equals $n$. In [2] Conner and Floyd determined the co-index of $\left(V\left(S^{n}\right), T_{2}\right)$ (for all $\left.n\right)$ and the co-index of $\left(P\left(S^{n}\right), T_{3}\right)$ for all $n$ except $n=2,4$, and 8. Their results assert (i) co-index $\left(V\left(S^{n}\right), T_{2}\right)=n$ or $n-1$ according as $n \notin\{1,3,7\}$ or $n \in\{1,3,7\}$ and (ii) co-index $\left(P\left(S^{n}\right), T_{3}\right)=n$ if $n \notin$ $\{1,2,4,8\}$ and $=n-1$ if $n=1$. The remaining cases of (ii) are resolved by

Proposition. For $n=2,4$, or 8 there exists an equivariant map $\left(P\left(S^{n}\right), T_{3}\right) \rightarrow\left(S^{n-1}, T_{1}\right)$.

Proof. For $n=2,4$, and 8 there are the Hopf fibrations $S^{n-1} \rightarrow$ $S^{2 n-1} \stackrel{p}{\rightarrow} S^{n}$. Here $S^{2 n-1}$ is the unit sphere in $F^{2}(F=$ complexes for $n=2$, the quaternions for $n=4$ and the Cayley numbers for $n=8)$ and the map $p$ assigns to each unit vector the 1-dimensional (over $F$ ) subspace it spans. Fix a point $x \in S^{n}$ and a point $y \in S_{x}^{n-1}=$ the fibre of $p$ over $x$. $S^{2 n-1}$ is the join of $S_{x}^{n-1}$ and $S_{-x}^{n-1}$, where $S_{-x}^{n-1}$ is both the fibre over $-x$ and the unit sphere in the 1-dimensional (over $F$ ) subspace orthogonal to the subspace spanned by $S_{x}^{n-1}$. Moreover $p$ maps the great circle arc

Received by the editors December 8, 1970.

AMS 1969 subject classifications. Primary 5536.

Key words and phrases. Fixed point free involution, equivariant map, co-index. 
[xz] for any $z \in S_{-x}^{n-1}$ 1-1 onto a great circle arc connecting $x$ and $-x$; as $z$ varies over $S_{-x}^{n-1}$ every great circle arc connecting $x$ to $-x$ is realized in the image of $p$ and $p\left[x z_{1}\right] \cup p\left[x z_{2}\right]$ forms a complete great circle passing through $x$ and $-x$ if and only if $z_{1}$ and $z_{2}$ are antipodal points of $S_{-x}^{n-1}$. Thus there is a 1-1 map $\varphi: S^{n} \rightarrow S^{2 n-1}$ whose image is the union $\bigcup[x z]$ taken over all $z \in S_{-x}^{n-1}$. Now if $E: P\left(S^{n}\right) \times I \rightarrow S^{n}$ denotes the evaluation map, then the composite $\varphi E$ restricts to a map $P\left(S^{n}\right) \times$ $\{1\} \rightarrow S_{-x}^{n-1}$ which by the definition of $\varphi$ sends $(\omega, 1)$ and $\left(T_{3} \omega, 1\right)$ to antipodal points for all $\omega$. This completes the proof.

Corollary. The co-index of $\left(P\left(S^{n}\right), T_{3}\right)$ is $n-1$ if $n \in\{2,4,8\}$.

Proof. As noted in [2] the co-index $\left(P\left(S^{n}\right)\right)>n-2$ for all $n$, since there is an equivariant embedding $e:\left(S^{n-1}, T_{1}\right) \rightarrow\left(P\left(S^{n}\right), T_{3}\right)$ which when composed with any equivariant map $\left(P\left(S^{n}\right), T_{3}\right) \rightarrow\left(S^{n-2}, T_{1}\right)$ would provide a contradiction to Borsuk's result quoted above. The Corollary then follows from the Proposition.

\section{REFERENCES}

1. K. Borsuk, Drei Sätze über die n-dimensionale euklidische Sphäre, Fund. Math. 20 (1933), 177-190.

2. P. E. Conner and E. E. Floyd, Fixed point free involutions and equivariant maps. II, Trans. Amer. Math. Soc. 105 (1962), 222-228. MR 26 \#768.

Department of Mathematics, Syracuse University, Syracuse, New York 13210 